\title{
CMOS-APS Detectors for Solar Physics: Lessons Learned during the SWAP Preflight Calibration
}

\author{
A. De Groof • D. Berghmans • B. Nicula · J.-P. Halain • \\ J.-M. Defise · T. Thibert • U. Schühle
}

Received: 22 November 2007 / Accepted: 12 March 2008 / Published online: 30 March 2008 (C) Springer Science+Business Media B.V. 2008

\begin{abstract}
CMOS-APS imaging detectors open new opportunities for remote sensing in solar physics beyond what classical CCDs can provide, offering far less power consumption, simpler electronics, better radiation hardness, and the possibility of avoiding a mechanical shutter. The SWAP telescope onboard the PROBA2 technology demonstration satellite of the European Space Agency will be the first actual implementation of a CMOS-APS detector for solar physics in orbit. One of the goals of the SWAP project is precisely to acquire experience with the CMOS-APS technology in a real-live space science context. Such a pre-
\end{abstract}

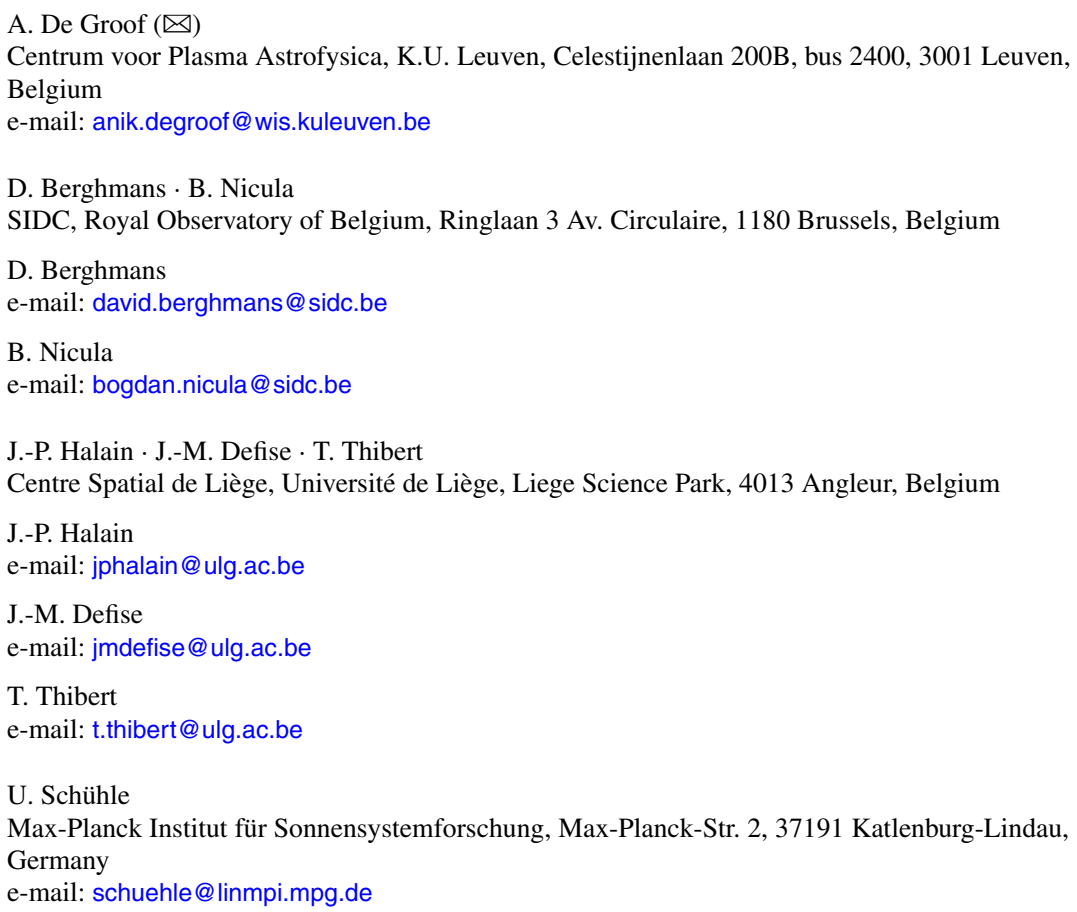


cursor mission is essential in the preparation of missions such as Solar Orbiter where the extra CMOS-APS functionalities will be hard requirements. The current paper concentrates on specific CMOS-APS issues that were identified during the SWAP preflight calibration measurements. We will discuss the different readout possibilities that the CMOS-APS detector of SWAP provides and their associated pros and cons. In particular we describe the "image lag" effect, which results in a contamination of each image with a remnant of the previous image. We have characterised this effect for the specific SWAP implementation and we conclude with a strategy on how to successfully circumvent the problem and actually take benefit of it for solar monitoring.

Keywords Instrumental effects · Spectrum: Ultraviolet

\section{Introduction}

Just like CCDs, CMOS-APS detectors are semiconductor arrays in which free electrons are generated under illumination through the photoelectric effect. The crucial difference between CCDs and CMOS-APS devices is in the way this collected charge is transferred from each pixel towards an analog-to-digital converter. For most CCDs, the charge is transferred over the sensor array, shifted from pixel to pixel until the charge is collected at a common readout port, which converts the charge to voltage and sends it off-chip. Instead, for CMOSAPS detectors, every pixel has its own readout port and the need for charge transfer is thus avoided. A clear disadvantage of the CMOS-APS detector is the fact that part of the photosensitive pixel area is sacrificed to accommodate the transistors required for the readout. This disadvantage is however offset by a number of benefits of CMOS-APS technology. While charge is being transferred, the CCD sensor array must be blocked from further illumination with a shutter to avoid image smearing. Since CMOS-APS detectors do not involve charge transfers, they do not require a mechanical shutter mechanism. Moreover, the charge transfer process requires significant power and is prone to radiation-induced aging. As a consequence, CMOS-APS detectors are far less power hungry and can be radiation hardened relatively easily. Another advantage of CMOS detectors is the high resistance to blooming. Finally, CMOS image sensors can also be more easily integrated into digital electronics than CCD devices, meaning that a CMOS-based camera can be significantly more compact than a comparable CCD camera.

For all these reasons, CMOS devices are becoming more popular for space applications. Compact CMOS cameras have been used for star trackers, for monitoring of robotic applications, and during docking maneuvers at the ISS and are beginning to be used for highdemanding scientific applications. In space-born solar physics, CMOS-APS detectors haveto the best of our knowledge - up till now only be used on the EUNIS sounding rocket (Rabin et al., 2003; Brosius, Rabin, and Thomas, 2007). Nevertheless, the upcoming Solar Orbiter mission, operating in a harsh radiation environment and facing severe mass constraints, does require CMOS-APS detectors for several of its instruments. It is thus important that the characteristics and possibilities of this type of detector become better known in the solar community. This is precisely one of the goals of the SWAP telescope (see Berghmans et al. (2006), and Defise et al. (2007), for a description of the early phase of the project) onboard the PROBA2 mission, and as well of the current paper.

PROBA2 is a microsatellite developed as an ESA technology mission by Verhaert Space (Kruibeke, Belgium) and is scheduled for launch towards the end of 2008. The PROBA2 mission is twofold. On one hand, it is a technology demonstration mission, testing new technologies for satellites and systems in-flight. On the other hand, PROBA2 has a scientific 
mission: Its Sun-synchronous orbit, combined with two instruments for solar observation SWAP and LYRA (Hochedez et al., 2006) - and two space weather instruments - DSLP and TMPU - forms the ideal basis for operational space weather forecasting.

The Sun Watcher with APS detector and image Processing (SWAP) will image the EUV Sun on the $1024 \times 1024$ pixels of a radiation-hardened CMOS detector. SWAP has two image-acquisition modes: Double Sampling (DS) and Correlated Double Sampling (CDS), corresponding to two strategies to remove the pixel offset (Section 2). In DS the output signal is produced on the sensor chip from the (analog) difference between the pixel voltage at the end of the integration and the reset voltage immediately following the integration. In CDS the pixel is read out twice, nondestructively: once in the beginning of the integration process and once at the end of the integration process. The difference then is produced off-chip after each sample has been digitised separately.

SWAP will image the Sun in a bandpass centred around $17.4 \mathrm{~nm}$ and optimised for capturing all space weather related solar events. The spectral selection is achieved with a set of aluminum foil filters, together with EUV reflective multilayer coatings deposited on the two mirrors of the Ritchey-Chretien off-axis telescope.

To understand the effects of the CMOS-APS detector and the compact optical design on the SWAP images, a preflight calibration was set up at the PTB-BESSY facilities in Berlin (Germany) in February 2007 (http://www.bessy.de). The aim of the calibration was to provide an adequate characterisation of the SWAP flight model for its scientific use. The EUV signal measured by SWAP was compared with the signal measured by a calibrated optical system. We collected measurements for the end-to-end radiometric calibration of the whole SWAP optical bench, which is composed of the entrance filter, the primary mirror, the secondary mirror, the rear filter near the focal plane assembly (FPA), and the detector. In addition, measurements for the characterisation of the spectral bandpass and the stray-light performance were undertaken.

Most of the calibration measurements were taken both with the DS and with the CDS image-acquisition modes. During the data-reduction process we soon realised that the two types of images differed in a subtle way and that in particular the DS images produced unexplained signal levels at low exposures. The reasons for these differences turned out to be rooted in the CMOS-APS technology. Depending on how the pixel reset function is implemented, images can be contaminated by remnants of the preceding image. It is clear that a complete understanding of this "image lag effect" is required for any quantitative use of CMOS-APS detectors in an astrophysical context. With the current paper we want to describe these CMOS-APS phenomena from the perspective of their use in the context of the SWAP solar telescope. The full instrument calibration and performance characterisation of the SWAP telescope depends on a complete understanding of the image lag phenomena and is therefore postponed to an upcoming paper. In what follows (Section 2) we give a detailed description of the two SWAP acquisition modes and link it with the electronic processes in each CMOS-APS pixel. After that we review our test setup at the PTB/BESSY facilities (Section 3), present our key measurements, and identify unexplained behaviour (Section 4). In Section 5 we explain our findings in terms of known effects in the CMOS-APS literature, applied to the specific case of the SWAP CMOS-APS detector. Finally, in Section 6 we summarise and conclude with the impact of the image lag effect on the SWAP operations and its potential benefit for solar monitoring. 


\section{CMOS Pixels and SWAP Acquisition Modes}

The CMOS detector used in the SWAP telescope is the High Accuracy Star tracker (HAS) ${ }^{1}$ developed for ESA by Fillfactory (Mechelen, Belgium, now part of Cypress). HAS is the follow-on of the more widely known STAR1000, featuring, among others, improved dark current and noise performance. The sensor consists of a square array of $1024 \times 1024$ three-transistor pixels, each measuring $18 \times 18 \mu \mathrm{m}$. As discussed by Janesick (2004) and illustrated by Figure 1, photodiode (PD) APS pixels comprise four major components. The photodiode region is responsible for generating and collecting signal charge. Three MOSFET transistors are required to measure the charge on the diode: $\mathrm{T} 1$ is a reset MOSFET, which resets the photodiode before charge is integrated on the diode, T2 is a source follower MOSFET, which converts signal charge to an output voltage, and T3 is a row-select MOSFET, which selects a pixel for readout (Pain et al., 2004; Janesick, 2004).

The readout process is done in three steps, as illustrated in Figure 2.

1. Activating the reset switch drains the (negative) charges present on the pixel's photodiode, corresponding to a black, preexposure state, or high voltage ( $V_{\mathrm{DD}}$, yellow in Figure 2).

Figure 1 Schematic architecture of photodiode CMOS-APS pixels.
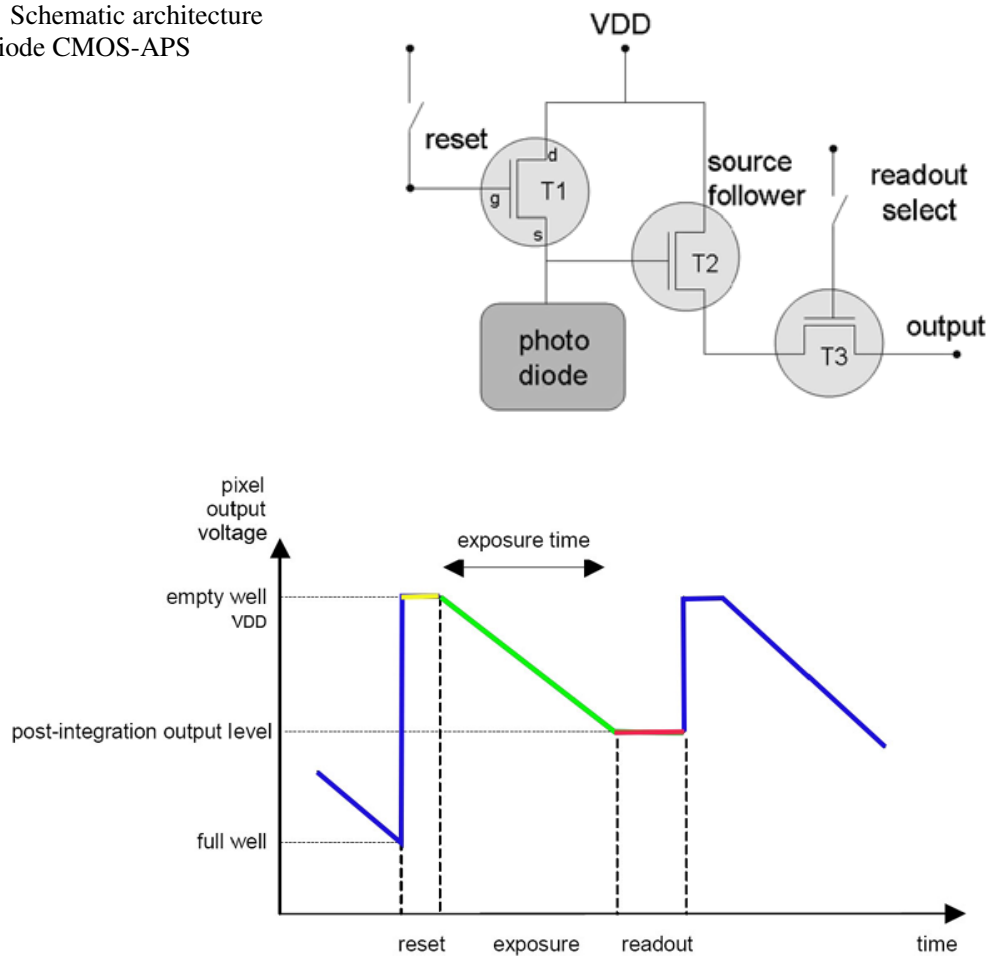

Figure 2 The signal lifetime in the pixel: reset to black level (high voltage $V_{\mathrm{DD}}$ : yellow), photocharge integration (dropping voltage: green), and voltage readout (red).

\footnotetext{
${ }^{1}$ This device is protected by U.S. patent 6225670 and others.
} 
2. During the exposure time (reset and select switch disabled) the pixel gathers (negative) charges on its photodiode caused by the impinging photons. During integration the voltage in the pixel decreases (green in Figure 2).

3. When the select switch is asserted the voltage on the pixel capacitor is connected to the pixel output and the pixel charge is "read out" (red in Figure 2).

Image acquisition is done by sequencing over all lines of interest and applying the required reset and/or readout select control to each line selected.

Based on these pixel electronics and readout procedure, two image-acquisition modes have been implemented for SWAP, which correspond to two strategies to remove the pixel offset:

- In Double Sampling the output signal is produced from the difference between the pixel voltage at the end of the integration and the reset voltage immediately following the integration. The scheme in Figure 3 (left) illustrates this process for five images with varying exposure times. The blue parts illustrate the voltage decrease during the exposure, the green dots mark the instances when the pixel is read out, and the red dots point to resets. The signal is calculated by subtracting the reset voltage after the image acquisition from the signal read after exposing the pixel (i.e., the voltages corresponding to the red and green dot at time $i$ are subtracted from each other to get signal $S_{i}$ ). It is important to note that DS is performed on-chip: The analog values are subtracted and only then converted to a digital signal by the 12-bit ADC.

- In Correlated Double Sampling the pixel is read out twice nondestructively, in the beginning and at the end of the exposure (i.e., the two green dots along the same blue line are subtracted from each other in the scheme of Figure $3 \mathrm{~b}$ ). Both values are sent to the output amplifier and through the ADC. The ADC digitises both signals to two 12-bit numbers, which are then subtracted in the camera electronics.

An implicit assumption in the DS mode is that the reset level is perfectly reproducible: It is assumed that the reset level following the exposure is exactly the same as the reset level preceding the exposure. Any fluctuation on the reset level will impact directly the measured signal. CDS does not have this reset noise problem, but in this case the quantisation noise is enhanced by a factor of $\sqrt{2}$ as a penalty for digitising before subtracting. For later reference it is important to point out that this difference between DS and CDS persists even in the
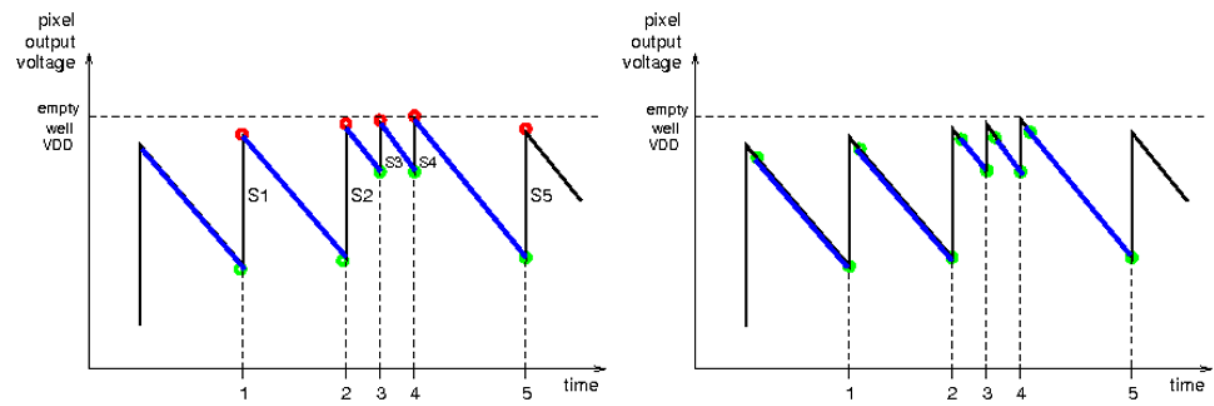

Figure 3 The readout process in both image-acquisition modes, Double Sampling (left) and Correlated Double Sampling (right), for a sequence of five images with different exposure times (and incomplete reset). Blue lines in the scheme on the left indicate the effective signals in DS mode; $S_{n}$ are the measured signals. Blue lines in the scheme on the right depict the signals measured in CDS mode. Red dots point to reset operation; green dots are readout operations. 
more extreme case of a incomplete reset, where the pixel is not fully drained after a strong exposure (as is suggested in Figure 3): DS images will be contaminated by whatever signal the reset could not remove, whereas in CDS such remnant signal drops out while subtracting.

\section{Calibration Test Setup}

The SWAP preflight calibration was set up at the PTB/BESSY facilities in Berlin (Germany) in February 2007 (http://www.ptb.de). A monochromatic EUV beam of the PTB beamline, generated at the storage ring BESSY II, was sent through the SWAP instrument and the signal caught on the detector was measured and compared to the optical system of PTB calibrated by a reference detector. We performed an end-to-end radiometric calibration of the whole SWAP instrument.

The SWAP instrument and the electronics box were fixed inside the PTB vacuum chamber and aligned with the EUV beam. Once under vacuum, the SWAP sensor was cooled to $-2^{\circ} \mathrm{C}$, a temperature close to the expected nominal in-flight conditions. After stabilisation of the temperature, the instrument door was opened and the calibrated EUV beam was measured by the SWAP detector.

The calibrated EUV beam of the PTB beamline is formed by a grazing incidence monochromator, fed by synchrotron radiation. At $17 \mathrm{~nm}$ the intensity is approximately $10^{10}$ photons per second and the standard shape of the beam is close to $1 \mathrm{~mm}^{2}$. Figure 4 (left) shows one of the first images of the EUV beam, adjusted to its maximal size while maintaining the homogeneity of the spot.

As clearly seen in the enlarged spot image of Figure 4 (right), the intensity is relatively inhomogeneous and that will complicate the analysis of the calibration results. A second complication, which is even more obvious, is the shadow effect of the rear filter grid (the filter closest to the detector). Because of the narrow and nearly collimated beam, the grid shows up much more clearly in this test than will be the case with a solar image. The conical beam in the test is so narrow that it is of the order of the grid rods in the filter plane. As a consequence, it is nearly fully blocked when on a filter rod, producing an important detector modulation. In flight conditions, with solar illumination, the full entrance aperture will be
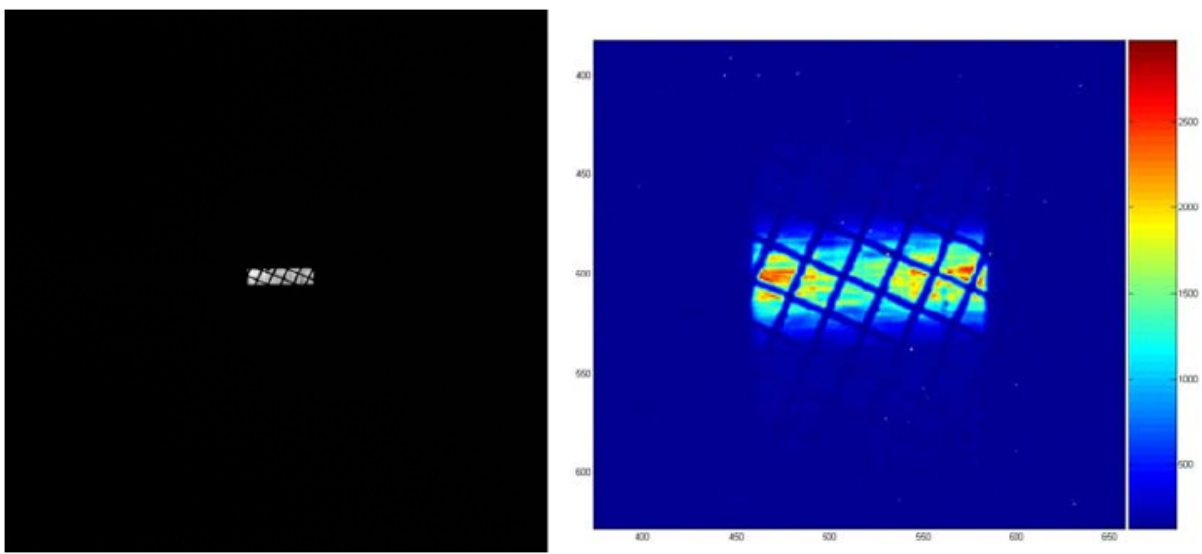

Figure 4 SWAP detection of the PTB/BESSY EUV beam. (Left) Full FOV. (Right) Detail in colour coding. 
illuminated and the light spot will have a diameter that is much larger than the rod size, making the modulation much less significant.

During the calibration campaign at PTB/BESSY the sensitivity and performance of the SWAP flight model were rigorously measured in several calibration tests. The measurements that are most important for understanding the side effects of the HAS detector and the two acquisition modes are (1) the noise evaluation and (2) the radiometric calibration with varying exposure time. In the following section we discuss the setup of these calibration tests and the measured differences between the DS and the CDS acquisition modes. The full instrument calibration and performance characterisation of the SWAP telescope, which depends on the results of the present study, will be published in a subsequent paper.

\section{Measurements}

\subsection{Noise Evaluation in Dark Images}

Right after the closing and vacuum pumping of the PTB chamber, the SWAP sensor was cooled from a temperature of 302.5 to $272 \mathrm{~K}$. During this period of almost 4 hours, "dark images" (with no EUV beam or visible light) were taken in both acquisition modes and with two integration times, 1 and $10 \mathrm{~s}$.

After the detector temperature was stabilised at $271 \mathrm{~K}\left(-2^{\circ} \mathrm{C}\right), 10$ series of dark images were taken with integration times of $1,2,3,4,5,7,10,14,18$, and $22 \mathrm{~s}$, respectively. Each series consists of 20 images. All these dark images help us to understand the noise in SWAP images. Although both acquisition modes "automatically suppress the flatfield" and "significantly reduce readout noise" (and reset noise in the CDS mode), this correction does not suppress $100 \%$ of the offset nonuniformity.

The measurements taken during cooling down show that the signal captured in a dark image decreases when temperature decreases, both for DS and CDS. This is an expected result since the dark current in the detector increases exponentially with temperature. The dark current is the signal that naturally occurs through the thermal generation of minority carriers in semiconductors. Figure 5 (top) shows the variation of the pixel mean as a function of temperature for images taken with CDS. Time runs from right to left in the top figure.

For images taken in the DS mode, however, the results show an additional and remarkable effect. In Figure 5 (bottom) the pixel mean of 1- and 10-s dark images is plotted against image number. The red curve depicts the temperature evolution. The sequence was built up as follows: The first five images were taken with 1-s integration time and the following five images with 10-s integration time. After a 10-min break (which explains the temperature jumps in the plot), the sequence was started all over again for another 14 times, until the final temperature was reached. The first images of each set seem to show a remarkable trend, with the signal measured in these dark images being offset from the signal in the rest of the image series. The offset seems to be related to the signal measured in the previous image. The plot shows that when a 1-s image follows a 10-s image (e.g., in image number 10), the signal is biased towards larger values. When a 10-s integration follows a 1-s integration (e.g., in image number 15), the measured signal is too low.

This "memory effect" was noticed while the calibration test was running and as an attempt to minimise this effect, two dummy images ${ }^{2}$ were inserted before each saved image.

\footnotetext{
${ }^{2}$ A dummy image is an image that is acquired in the same way as a regular SWAP image but is not saved.
} 


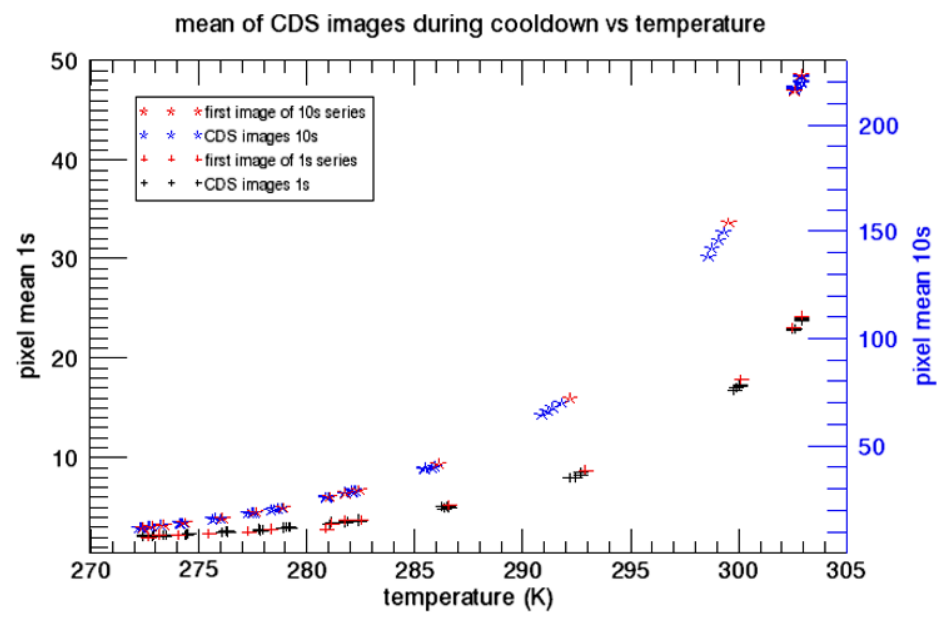

Mean of $1 \mathrm{~s}$ and 10s DS images during cooldown

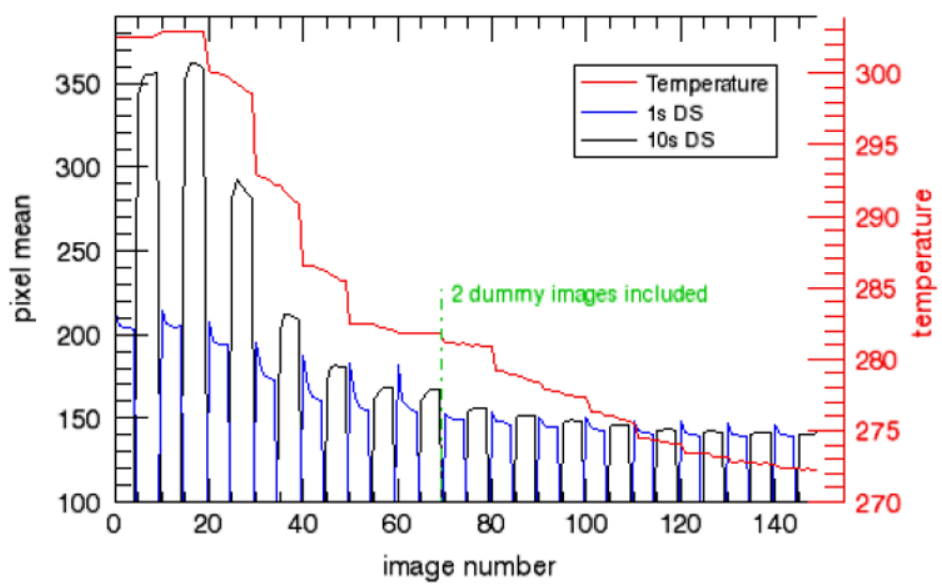

Figure 5 (Top) Pixel mean in CDS images as a function of temperature during the cooling phase. Red points are associated with the first image of each set. (Bottom) Temperature evolution and pixel mean in DS images as functions of image number (chronological in time).

In this way extra resets are built in and the influence of a previous image with different exposure time is decreased. Starting from image number 70 (pointed out by the green line in Figure 5 bottom), we followed this new strategy and the effect is clearly visible in the plot: The memory effect is much less obvious although still visible in the evolution of the pixel mean. Images taken in the CDS mode, however, do not show this effect at all: The pixel mean of the first image in a set is completely consistent with the following images taken with the same integration time (see Figure 5 top). More tests with varying exposure time (during EUV illumination) were done during the radiometric calibration, which is described in Section 4.2.

From the dark images taken at a stabilised temperature, $271 \mathrm{~K}$, three kinds of noise were evaluated: fixed pattern noise, dark current (DC) rate, and dark noise. The fixed pattern is the residual offset still present in all pictures and is calculated as the average of several pictures at very short integration time. The fixed pattern noise is the standard deviation 
Table 1 The noise measured during the calibration tests, at $-2^{\circ} \mathrm{C}$, for the two acquisition modes, DS and CDS. (The $e^{-} / \mathrm{DN}$ conversion factor used is $\left.20 e^{-} / \mathrm{DN}.\right)$

Figure 6 Histogram-equalised images of the beam in location 1 . The red box is the location of the spot; the green box is the location of the dark pixels analysed in the text.

\begin{tabular}{lcc}
\hline & DS mode & CDS mode \\
\hline Fixed pattern noise $\left(e^{-}\right)$ & 32 & 3 \\
Mean dark current rate $\left(e^{-} / \mathrm{s}\right)$ & 4.5 & 19.9 \\
Dark noise $\left(e^{-}\right)$ & 42.2 & 34
\end{tabular}

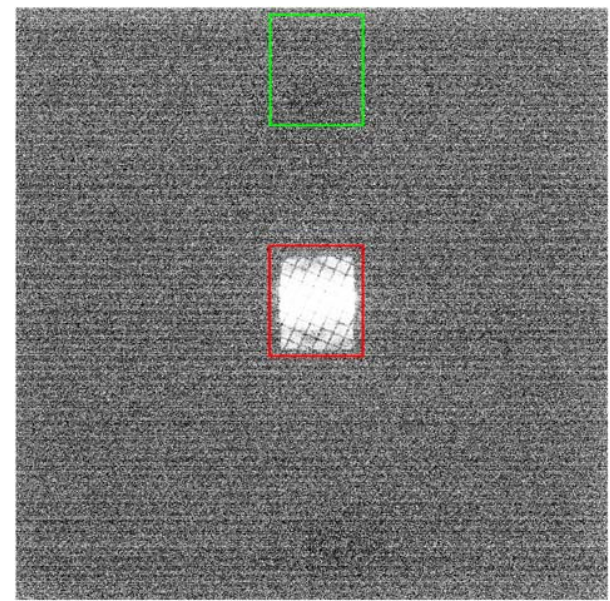

of the fixed pattern. To characterise the dark current $(D C)$ rate, average dark images (i.e., averaged over several identically acquired dark images) with different integration times are constructed. The scatterplot of the pixel means (i.e., mean of all pixel values in an image) of these average dark images as a function of the integration time can be fitted linearly. The slope of these fits gives a value for the DC rate. The dark noise, finally, is the temporal noise (a measure of the pixel behaviour in time) at very short integration time. In Table 1, the results found during the calibration tests $\left(\right.$ at $-2^{\circ} \mathrm{C}$ ) are summarised.

As mentioned before in Section 2, both acquisition modes are designed to reduce readout noise but the CDS mode should do even better since reset noise is also cancelled out. The noise evaluation results indeed show a lower fixed pattern noise and dark noise for the CDS mode compared to the DS mode. However, the CDS mode seems to have a higher dark current rate. The reason for this apparent inconsistency is discussed in Section 5.

\subsection{Radiometric Calibration and Instrumental Response}

After the noise evaluation in dark images was done, the SWAP door was opened and the BESSY II EUV beam was measured at the SWAP CMOS detector. The radiometric calibration was performed as a function both of exposure time as well as of beam intensity. The first test, in which sets of images with different exposure times were acquired in a random order, is particularly instructive for studying the memory effect in the DS mode. Eleven series of 20 identically acquired images were taken with the following exposure times: 4, 14, 1, 22, 7 , $18,2,10,3,5$, and $4 \mathrm{~s}$ again for comparison with the first series. This sequence was repeated at two locations on the detector, and for the two readout modes.

Figure 6 shows a brightness-enhanced image of the beam in the first location. To investigate the memory effect, we study the evolution of the mean pixel value in the 220 images of 

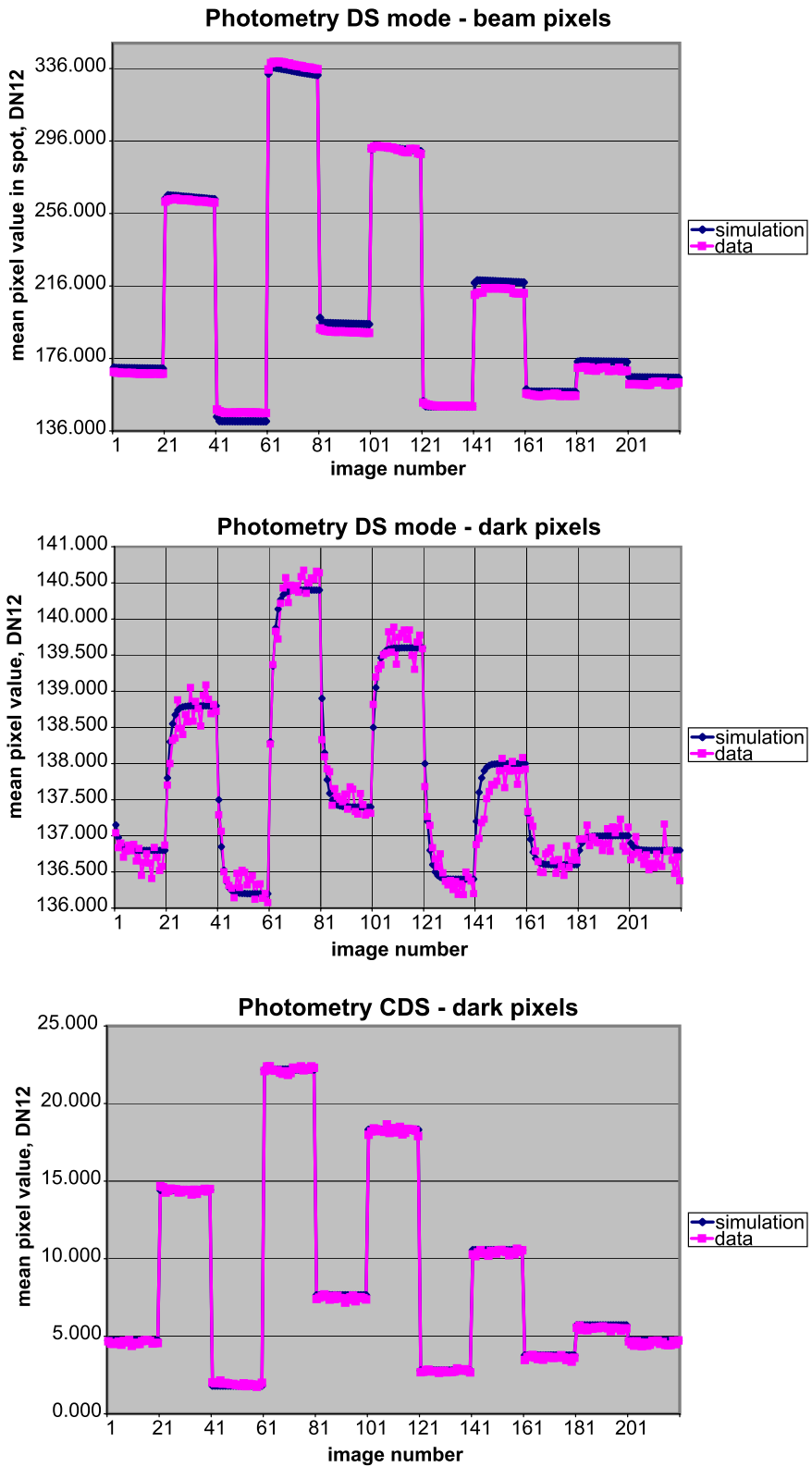

Figure 7 (Top) Mean values of the beam pixels (red in Figure 6) in DS data of the radiometric calibration data. The simulation takes into account a ghost of the previous image of $2 \%$. (Middle) Mean values for the dark pixels (green in Figure 6). The simulation is based on a ghost of 50\% of the previous image. (Bottom) Mean values of the dark pixels in CDS data.

the radiometric calibration data. Averaging over the whole image would increase noise, so we first study the so-called beam pixels, defined as the pixels inside the red box of Figure 6 . Figure 7 (top) shows, in pink, the mean values of the beam pixels throughout the sequence. 
An overall decline in mean pixel value is seen throughout the test but this effect is due to the decreasing storage ring current of Bessy II and has nothing to do with the HAS detector. It also explains the difference between the first and the last plateaus. Apart from this, one can see that the first data pair of each plateau is biased towards the previous plateau. This memory effect can be simulated in a first attempt by taking into account a "ghost" of $2 \%$ of the signal in the previous image (blue). Note however that the effect is not that obvious as in the dark images taken in the DS mode during the cooling period (see Figure 5 bottom). To verify this, we made a similar plot for the dark pixels in the image, defined as the green box in Figure 6. The result is shown in Figure 7 (middle) and the conclusion is clear: The memory effect is much more important in "dark pixels" than in "beam pixels." The simulation of the dark-pixel signals (in blue) is based on a ghost of 50\% of the previous image! This was, at first, a surprising and unexplained effect, especially since none of this is seen in the CDS images (see Figure 7 (bottom)). Further investigations, however, revealed that the reason for this difference is rooted in the CMOS-APS technology, as we will discuss in Section 5.1.

Another result that was inferred from the radiometric calibration with variation in exposure time is the instrumental response of SWAP. The results are summarised in Figure 8, for the two acquisition modes (DS at the top and CDS at the bottom). From this figure we can deduce the average performance of SWAP: $1145 \mathrm{ph} / \mathrm{DN}$ for DS and $981 \mathrm{ph} / \mathrm{DN}$ for CDS. But in the framework of the present paper we are most interested in the different slopes of the SWAP response in the two acquisition modes. For both positions on the detector, the slope of the response in the DS mode is clearly nonlinear for small exposure times (and thus small signals). The CDS curve, however, shows a very linear behaviour. A discussion of whether and how this is related to the earlier mentioned memory effect is given in Section 5.

\subsection{Tracing the Memory Effect in Individual Images}

If the memory effect is indeed caused by a ghost of the previous image, as suggested in the previous section, we should see some sign of it in the images themselves. DS images do not show any obvious sign of a ghost when inspected with the naked eye. However, brightnessenhanced images can help to reveal the real problem. Figure 9 shows a histogram-equalised image, caught in the DS mode with the bright, rectangular beam spot located at the bottom of the image. Ghost remnants can be seen from the previous beam spot position (at the top of the image) and from the path between the previous and the current spot locations (vertical stripe). As SWAP does not have a shutter, the detector is exposed during the whole period in between image acquisitions and the movement of the beam is registered as well.

\section{HAS as a CMOS Photodiode}

\subsection{Image Lag}

The so-called memory effect appearing in the DS mode turns out to be inherent in the design of the SWAP pixels. The SWAP CMOS pixels are PD APS pixels (Figure 1), which are reset using the "soft reset" mode of the HAS sensor. The PD pixel has many desirable qualities, including high quantum efficiency, good fill factor, low cross-talk for visible wavelengths, high full well, and anti-blooming. But high reset noise is a weakness. ${ }^{3}$ The soft reset mode is used to reduce this reset noise but this is achieved only at the expense of higher image lag and a markedly nonlinear response under low illumination. The reasons for these side effects are explained in the following.

\footnotetext{
${ }^{3}$ This reset noise is significantly reduced when one applies CDS in the NDR mode.
} 

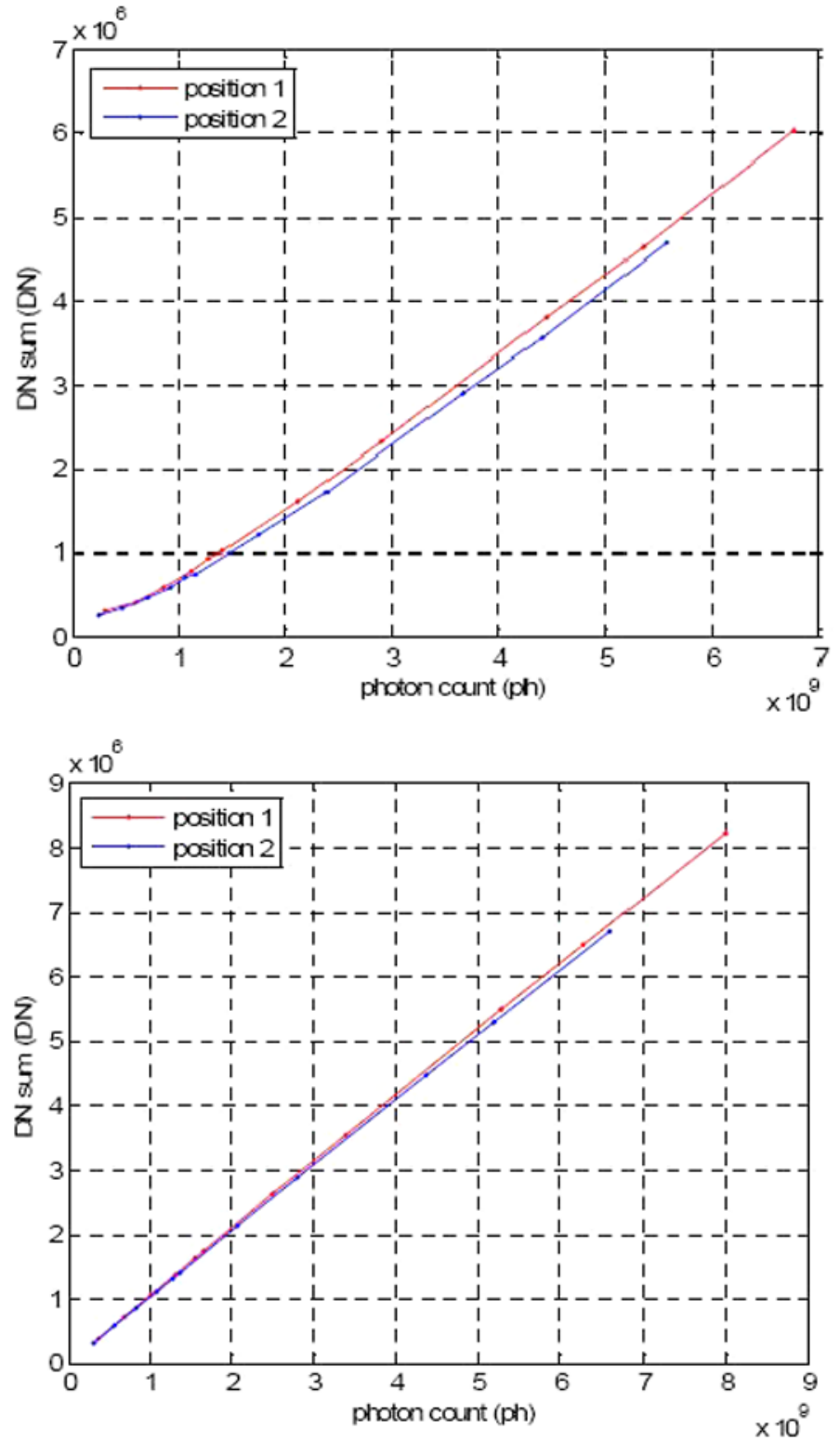

Figure 8 The instrumental response measured using different exposure times. The results for DS are shown in the top plot and those for CDS in the bottom plot.

The reset transistor (T1 in Figure 1) is responsible for resetting the pixel and has three pins: the source (lower side, "sense node" connected to the photodiode), the gate (left side, connected to the reset voltage), and the drain (upper side, connected to the power supply voltage $\left.+V_{\mathrm{DD}}\right)$. The functioning of the reset transistor depends on the voltage difference between gate and source $\left(V_{\mathrm{GS}}\right)$, the voltage difference between drain and source $\left(V_{\mathrm{DS}}\right)$, and their relation to $V_{\mathrm{th}}$, the intrinsic threshold voltage of the transistor. This threshold voltage 
Figure 9 A SWAP image, taken in the DS mode, with the bright, rectangular beam spot at the bottom of the image. Ghost remnants can be seen from the previous beam spot position (top) and from the path between the previous and the current spot locations. The image is histogram equalised to enhance the weak ghost contributions; in the original image the ghost could not be seen.

Figure 10 Cross section of an n-channel MOSFET.
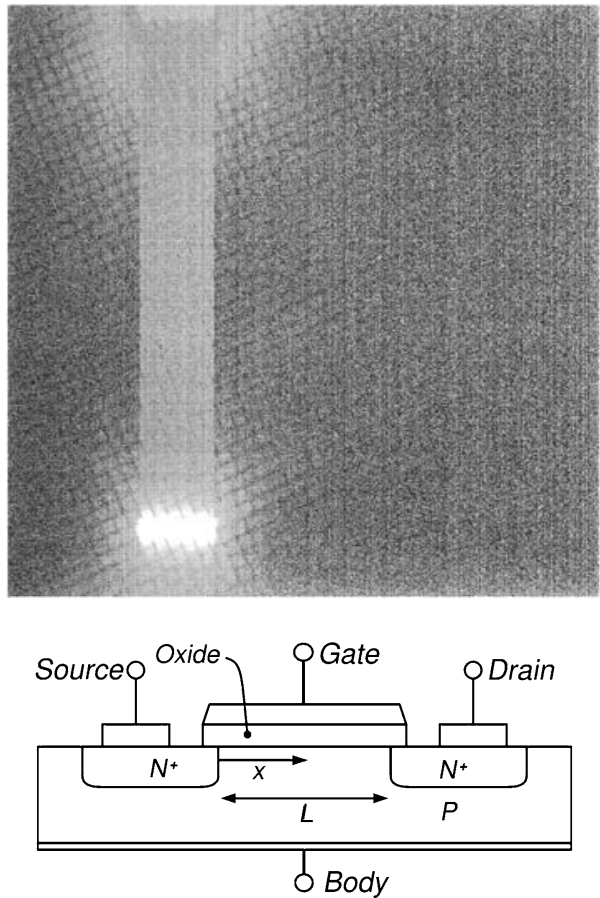

depends on how strong the substrate is $p$-doped, or, in other words, the excess of electron holes under the gate. When a positive voltage is applied on the gate, electrons in the substrate accumulate in the region of the substrate nearest to the gate and fill the electron holes, creating a depletion region. This $n$-channel extends between the source and the drain (length $L$ in Figure 10), but current is conducted through it only when the gate potential is high enough to attract electrons from the source into the channel. When zero or negative voltage is applied between gate and source, the channel disappears and no current can flow between the source and the drain.

Figure 11 shows three different regimes in which the reset transistor can work, depending on the value of $V_{\mathrm{GS}}$ :

1. $V_{\mathrm{GS}}<V_{\mathrm{th}}$ (cutoff or subthreshold regime): If the voltage between gate and source, $V_{\mathrm{GS}}$, is smaller than $V_{\text {th }}$, then according to the basic threshold model, the transistor switches off, and there should be no conduction between drain and source.

In reality, the Boltzmann distribution of electron energies allows some of the more energetic electrons at the source to enter the channel and flow to the drain, resulting in a subthreshold current that is an exponential function of gate to source voltage. Although the current between drain and source should ideally be zero when the transistor is in subthreshold mode, there is a weak-inversion current, sometimes called subthreshold leakage.

2. $V_{\mathrm{GS}}>V_{\mathrm{th}}, V_{\mathrm{GS}}<V_{\mathrm{th}}+V_{\mathrm{DS}}$ (saturation regime): The transistor is turned on, and a channel has been created, however, only over a part of the length $L$ in Figure 10. This allows limited current to flow between the drain and source. The drain current is now quadratically proportional to $V_{\mathrm{GS}}$.

3. $V_{\mathrm{GS}}>V_{\mathrm{th}}, V_{\mathrm{GS}}>V_{\mathrm{th}}+V_{\mathrm{DS}}$ (triode or linear regime): The transistor is turned on and a full $n$ channel is created between source and drain. 
Figure 11 The three regimes in which a MOSFET transistor can be operated, depending on the value of $V_{\mathrm{GS}}$ : (1) cutoff or subthreshold, (2) saturation, and (3) triode or linear regime. VTH is the threshold voltage.

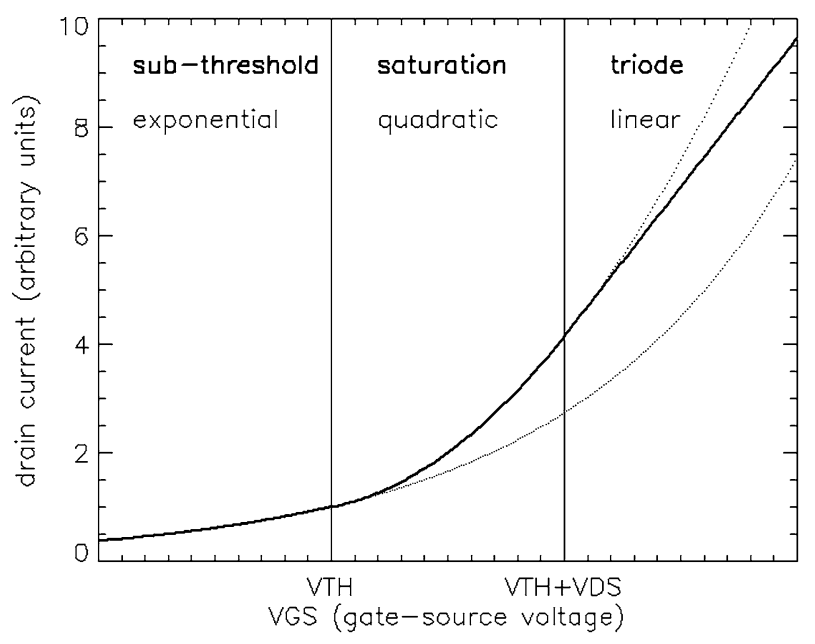

In "hard reset," the voltage applied on the gate is large such that $V_{\mathrm{GS}}$ is large and we are always in the triode or linear regime. The hard reset operation (in contrast to soft reset; see the following) has the advantages that the drain current is large, resulting in a fully reset pixel (no signal remnant), and that the depth of the full well is maximised. It has the disadvantage that it is a noisy operation. The voltage noise variance in a hard-reset (gatevoltage-regulated) pixel equals $\sigma=\sqrt{k T / C}$, where $C$ is the gate capacitance.

A second well-known mode to reset a CMOS PD pixel is called soft reset. In this case, the voltage applied to the reset gate is the same as the one applied to the drain node (i.e., $\left.+V_{\mathrm{DD}}\right)$. The voltage difference between gate and drain is then zero. This means that

$$
V_{\mathrm{GS}}=V_{\mathrm{GD}}+V_{\mathrm{DS}}=V_{\mathrm{DS}}<V_{\mathrm{DS}}+V_{\mathrm{th}} .
$$

For weakly exposed pixels that have collected only a small negative charge $\left(V_{\mathrm{GS}}<V_{\mathrm{th}}\right)$, the transistor operates in cutoff or subthreshold mode. Strongly exposed pixels $\left(V_{\mathrm{GS}}>V_{\mathrm{th}}\right)$ are reset in the saturation regime. In either case, the current from source to drain is limited, making it hard to fully reset the source (bring it up to $V_{\mathrm{DD}}$ voltage).

Soft reset is known (Pain et al., 2004) to be less noisy $(\sigma=\sqrt{k T / 2 C})$ than hard reset $(\sigma=\sqrt{k T / C})$, although actual hardware implementation details might complicate the comparison (e.g., differences in feed-through voltage). But soft reset has three clearly posed, related disadvantages:

- Owing to the subthreshold regime the reset is often incomplete. A remnant of the signal remains in the pixel at the beginning of the next exposure. This results in image lag.

- The subthreshold current (and thus the success of the reset) is an exponential function of gate-source voltage (i.e., of the signal in the photodiode). The success of the reset is thus dependent on the recorded signal: the larger (negative) the signal, the better the current and thus the reset.

- For small signals the response can be highly nonlinear up to the case that no subthreshold current is established at all, resulting in apparent signal loss.

\subsection{Linking Soft Resets with the Memory Effect}

The memory effect seen in the radiometric calibration data taken during the PTB/BESSY campaign can easily be linked to the effects associated with the soft resets in SWAP pixels. 


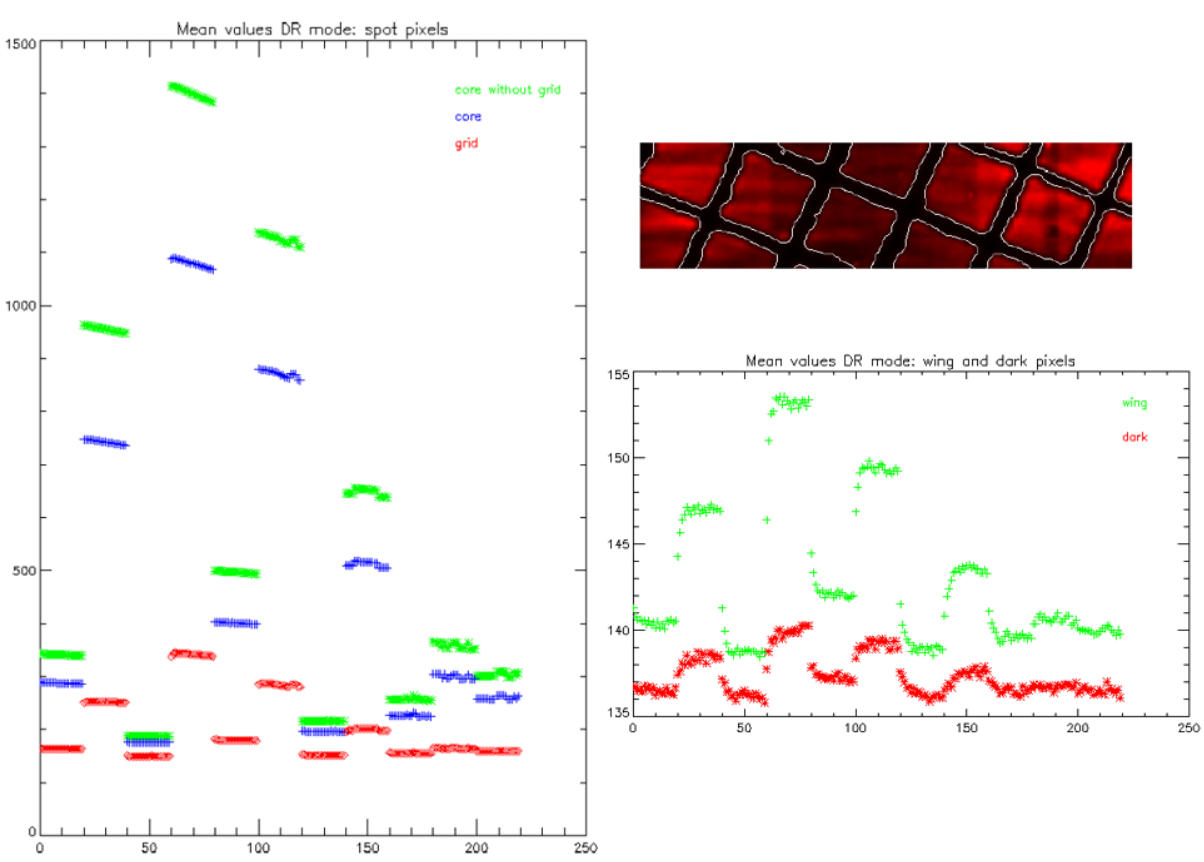

Figure 12 The mean pixel values in DS images of spot core and grid pixels (left) and of wing and dark pixels (right). The horizontal axis shows the image number in the complete image sequence. In the top-right image, a contour line shows the border between core and grid pixels.

As discussed in Section 4.2, the data series taken with varying exposure time showed that a DS image is influenced by the signal strength in the previous image: When switching from a long exposure time to a shorter one, the first images of the new series have a mean pixel value that is too high compared to the rest of the images in the series. In case a shorter exposure time preceded the actual image, the measured pixel values are too small. In CDS images, this trend is not seen, and all images with identical exposure times have very similar mean pixel values. This difference can be understood perfectly with the idea of incomplete resets since these only have an effect in the DS mode (see Figure 3).

To test whether this trend in the DS mode is due to the soft reset with nonlinear behaviour at small signals, the mean pixel values were plotted for different groups of pixels: spot core pixels, grid pixels (pixels that are darker because of the shadow of the grid rods), wing pixels (in which the beam spot has dark extensions above and under the spot core - see Figure 4b), and dark pixels. Figure 12 shows the mean pixel values in the DS mode for spot core and grid pixels (left) and for wing and dark pixels (right). The top-right image illustrates how we distinguished between core and grid pixels.

The plots unambiguously show the important difference in memory effect between weak and strong illuminated pixels. In CDS mode images, in contrast, even the least illuminated "dark" pixels do not show any effect at all. This important difference with the CDS mode, along with the strong proof of a nonlinear response for small pixel charges, leads to the natural conclusion that the "ghosts" seen in the DS mode are due to an incomplete, soft reset of the SWAP pixels.

The soft reset can also explain the lower dark current rate in DS images as compared to CDS images (see Section 4.1). At first this result was surprising since the dark current 
should be essentially the same in the two acquisition modes. However, because of the soft reset, part of the signal is lost in an incomplete readout of the pixels. Especially for lowsignal pixels, an important part of the signal, and also of the noise, is lost while reading out in subthreshold mode.

\section{Conclusions}

During the analysis of the preflight calibration data of SWAP, we have discovered an unexpected behaviour of the HAS detector: Seen in the images are "ghosts," which are remnants of the previous exposure. This effect, also called "image lag," is seen only in the Double Sampling acquisition mode (but not in the Correlated Double Sampling mode). It is small $(<1 \%$ of full well) and mostly present in weakly exposed pixels. It nevertheless clearly shows up in histogram-equalised images (see Figure 9).

Image lag is a known problem for CMOS-APS detectors and is due to an incomplete reset operation. The completeness of the reset depends on the voltage level applied to the gate of the reset transistor as compared to its intrinsic threshold voltage of the device. In "hard reset" the applied reset voltage is large enough to completely drain the pixel well. Applying a lower reset voltage ("soft reset," implemented for SWAP) improves the noise level of the resulting pixel state, but now the completeness of the reset is a function of the initial charge collected in the pixel: the larger the collected charge, the more complete the reset is. For weakly exposed pixels, the reset is incomplete and a remnant remains in the pixel of the pre-exposure state.

As SWAP does not have a shutter, this ghost image is actually an integration of all incident light on the detector since the last reset (of the preceding image). The ghost in the image thus gives us access to events happening in between two images. Image lag is not observed in the Correlated Double Sampling acquisition mode, as in this case the ghost is present in both terms of the differencing operation and thus cancels out. Therefore Correlated Double Sampling will be the nominal acquisition mode for SWAP and the image lag effect will not hinder the normal scientific functioning of the telescope.

Given the specific solar target of SWAP, the image lag in the Double Sampling mode is however more than just an annoyance and can be seen as a useful feature. Given its dependence on the recorded signal, image lag will only appear in the darkest areas of each image. For appropriately exposed solar images, this means the far off-disk region. The radiation from this part of the solar corona is usually too dark, in comparison with the on-disk region, to produce significant signal. The exceptions are transient events such as filament eruptions that transverse the far off-disk region at high speeds (typically a few hundred kilometers per second). These solar eruptions are difficult to image as their transit time through the field of view is typically much less than the time in between two image exposures (the cadence).

However, in the Double Sampling mode, such transient features will remain observable in the following image through the image lag effect. The observed signature will be smeared along the line of propagation but otherwise perfectly identifiable in histogram-equalised images such as Figure 9. These smeared propagation paths will clearly show the link between the source regions near the solar disk and coronal mass ejections observed in coronagraph images. Yet, as a characteristic of the image lag effect, the smeared transient events will only appear in the images where these images would have been dark, retaining the rest of the image (especially the disk region) uncontaminated. The image lag effect thus offers in principle great potential for the detection of solar eruptions and monitoring solar activity in general. We plan to test and demonstrate this potential in the early phase of the SWAP mission. 
Acknowledgements The SWAP instrument was developed by the Centre Spatial de Liège (Université de Liège, Belguim) in collaboration with the Royal Observatory of Belgium. The work at these institutes and at the K.U. Leuven is supported by PRODEX Grant No. C90196 (SWAP - Preparation to Exploitation), managed by the European Space Agency in collaboration with the Belgian Federal Science Policy Office (BELSPO). The SWAP calibration campaign was financially supported by the Max-Planck-Institut für Sonnensystemforschung, Germany, through collaboration with Physikalisch-Technische Bundesanstalt (PTB) at BESSY. It is a pleasure to acknowledge the discussions within the Science Consortium for SWAP and LYRA (http://proba2.sidc.be/SCSL/), held at the International Space Science Institute (Bern, Switzerland).

\section{References}

Berghmans, D., Hochedez, J.F., Defise, J.M., Lecat, J.H., Nicula, B., Slemzin, V., et al.: 2006, Adv. Space Res. 38, 1807.

Brosius, J.W., Rabin, D.M., Thomas, R.J.: 2007, Astrophys. J. 656, L41.

Defise J., Halain J., Berghmans D., Denis F., Mazy E., Rochus P., et al.: 2007, In: Fineschi, S., Viereck, R.A. (eds.) Solar Physics and Space Weather Instrumentation II, Proc. SPIE 6689, 66890S.

Hochedez, J.F., Schmutz, W., Stockman, Y., Schühle, U., Benmoussa, A., Koller, S., et al.: 2006, Adv. Space Res. 37, 303.

Janesick J.R.: 2004, In: Grycewicz, T.J., McCreight, C.R. (eds.) Focal Plane Arrays for Space Telescopes, Proc. SPIE 5167, 1.

Pain B., Yang G., Ortiz M., Wrigley C., Hancock B., Cunningham T.: 2004, In: 1999 IEEE Workshop on Charge-Coupled Devices and Advanced Image Sensors. http://hdl.handle.net/2014/17488.

Rabin, D., Davila, J., Thomas, R.J., Engler, C., Irish, S., Keski-Kuha, R., et al.: 2003, Bull. Am. Astron. Soc. 35, 845 . 